\title{
Educação superior a distância: fatores pre- ditores da evasão anteriores a admissão de estudantes
}

\section{Resumo}

Oberdan Santos da Costa

Luis Borges Gouveia

Universidade Fernando Pessoa (Portugal)

Baseado em estudos anteriores na área da evasão voluntária de estudantes adultos não tradicionais, o presente trabalho tem como objetivo identificar e compreender os fatores preditores da evasão voluntária de estudantes anteriores a admissão nas instituições de ensino superior a distância, tais como: sexo, idade, etnia, estado civil, entre outros. Objetivando maior proximidade com o universo desse estudo, adota-se a pesquisa do tipo exploratória, pois o método envolve o levantamento de trabalhos de tese, dissertação e artigos científicos na literatura nacional e internacional. Os resultados desse estudo revelaram que os fatores preventivos de evasão anteriores a admissão, incluindo idade, sexo, educação anterior, Grade Point Average GPA, números de cursos concluídos a distância e habilidades de gerenciamento de tempo são significativos para a prevenção da evasão voluntária de estudantes adultos e proporcionam informação para o desenvolvimento de intervenção precoce. Os resultados auxiliam também os pesquisadores a focar os seus estudos.

Palavras-chave: Educação a distância. Preditores. Evasão. Fatores. Admissão.

\section{Higher distance education: predictive factors for predatory admission to students}

\begin{abstract}
Based on previous studies in the area of non-traditional adult student's dropout, the present study aims to identify and understand the predictive factors of voluntary dropout of pre-admission student's factors for distance learning institutions such as sex, age, ethnicity, marital status, among others. To conduct the present research, an exploratory approach is adopted, involving the collection of thesis, dissertation and scientific articles both from national and international sources. The results of this study revealed that pre-admission prevention dropout factors, including age, gender, previous education, Grade Point Average GPA, distance completed course numbers, and time management skills are significant for preventing student's adults dropout, and provide information for the development of early interventions. It also can provide useful support to researchers and scholars in choosing the focus and purpose of their studies.
\end{abstract}

Keywords: Distance education. Predictors. Dropout. Factors. Admission. 
Educação superior a distância: fatores preditores da evasão anteriores a admissão de estudantes

\section{Educación superior a distancia: factores predictores de la evasión anteriores a la admisión de estudiantes}

\section{Resumen}

Basado en estudios previos en el área del abandono escolar no tradicional de estudiantes adultos, el presente estudio tiene como objetivo identificar y comprender los factores predictivos del abandono voluntario de los factores de estudiantes de pre-admisión para instituciones de aprendizaje a distancia como el sexo, la edad, el origen étnico, el estado civil, entre otros. Para llevar a cabo la presente investigación, se adopta un enfoque exploratorio, que incluye la recopilación de tesis, disertaciones y artículos científicos de fuentes nacionales e internacionales. Los resultados de este estudio revelaron que los factores de abandono de la prevención antes de la admisión, incluidos la edad, el sexo, la educación previa, el promedio de calificaciones, el número de cursos a distancia y las habilidades de gestión del tiempo son importantes para prevenir el abandono escolar de los estudiantes y proporcionan información para El desarrollo de intervenciones tempranas. También puede brindar un apoyo útil a los investigadores y académicos para elegir el enfoque y el propósito de sus estudios.

Palabras claves: Educación a distancia. Predictores. Evasión. Factores. Admisión.

\section{Introdução}

Nos últimos dez anos (2007-2016), presenciamos um considerável crescimento no sistema educacional da modalidade de ensino a distância no Brasil, com regime administrativo (público e privado), IES credenciadas, número de polos e novos cursos para atender uma demanda crescente de estudantes. De acordo com o Censo da Educação Superior de 2016, o Brasil conta com 217 IES credenciadas para ministrar a EaD, 5.133 polos de atendimento aos estudantes, 1.662 cursos na modalidade à distância e 1.494 .418 estudantes matriculados. Acrescente-se a esses dados, uma diversidade das regiões, critérios, contribuições das políticas públicas de acesso implementadas e processos seletivos de admissão para a eficiência interna dos programas, no sistema de educação do país (BRASIL, 2017).

Em referência as políticas públicas adotadas, estas refletem uma parte desse crescimento no sistema educacional, pois não acompanham, tão pouco atendem em sua totalidade as crescentes demandas de vagas, principalmente nas universidades públicas. 
Já no tocante aos processos seletivos de admissão no curso superior, as instituições vêm se adequando e melhorando conforme aumento no quadro de demanda por vagas. No entanto, essa "melhora na aplicação dos processos seletivos", principalmente nas instituições privadas, no que diz respeito a limitação de conteúdo dos processos seletivos de admissão tem implicado na perda da qualidade de alguns processos seletivos, deixando-os a desejar e sendo considerados ineficazes, pois não refletem o mínimo de preparação do estudante para entrar na graduação.

Pressupõe-se que um processo seletivo de admissão eficaz de estudantes é fundamental e importante em qualquer sistema de ensino superior, pois contribui para garantir que o crescimento da matrícula esteja relacionado com a capacidade de instrução do estudante, com a redução do quadro de evasão da instituição, com a qualidade da educação superior e com as perspectivas de futuros empregos, ganhos e crescimento econômico dos estudantes. Nesse sentido, Gillis (2007) sugeriu critérios de admissão mais rigorosos para os programas de graduação em ciências da saúde de uma instituição e afirmou que mudanças no processo de recrutamento e admissão podem ajudar a diminuir as taxas de evasão.

A evasão de estudantes tem sido citada por vários autores como um fenômeno complexo, porque envolve um comportamento humano que varia com o tempo (WOODLEY, LANGE, TANEWSKI, 2001 ; HOLDER, 2007). Esse problema afeta o estudante, as instituições (públicas e privadas) e também a sociedade. $\bigcirc$ problema do alto índice de evasão nos cursos de EaD tem motivado pesquisas sobre os fatores que contribuem para esse fenômeno. 0 Censo EAD.BR 2016 registrou uma evasão entre $11 \%$ a 25\%, com 32\% das ocorrências nas instituições que oferecem cursos regulamentados totalmente a distância (ASSOCIAÇÃ́O BRASILEIRA DE EDUCAÇÃ̂ À DISTÂNCIA, 2017), mesmo com taxas menores em relação ao Censo EAD.BR 2015, ainda é muito preocupante, tendo em vista que a quantidade de matriculas dos estudantes de 2016 também foi menor em relação a de 2015. Ainda sobre o Censo EAD. BR 2016 e segundo os respondentes, os principais motivos que ocasionam a evasão são os motivos financeiros e de tempo.

Crescimento na população universitária da modalidade de ensino a distância, tem feito surgir uma diversidade de fatores, que parecem não estar sendo identificados e compreendidos pelas instituições. De acordo com Holder 
Educação superior a distância: fatores preditores da evasão anteriores a admissão de estudantes

(2007) os passos pelos corredores da academia estão sendo rapidamente substituídos por toques no teclado do ciberespaço.

Nesse sentido, a não compreensão dos fatores anteriores a admissão de estudantes pode implicar na evasão precoce voluntaria de estudantes gerando perda para todos envolvidos no processo da educação. Segundo Abbad, Carvalho e Zerbini (2006, p. 6) "[...]a falta de informações sobre o perfil de entrada dos participantes quanto às suas características motivacionais, cognitivas, demográficas e profissionais, hábitos e estratégias de aprendizagem [...]" afetam desfavoravelmente o desenho adequado de cursos na modalidade a distância. Monereo e Pozo (2010) vão um pouco além dessa compreensão, eles realizaram uma contextualização do que se espera dos alunos que estão ingressando nas escolas e quais as competências envolvidas no processo. Os autores analisaram o cenário social e principalmente quem são os nativos e imigrantes digitais'.

Assim, o novo contexto do cenário educacional marcado pelo crescimento da população de estudantes adultos levou as instituições a experimentarem uma mudança demográfica à medida que os estudantes significativamente mais velhos estão entrando e voltando à educação pós-secundária. Tais mudanças trouxeram uma ênfase maior às necessidades particulares, características, estilos de vida, motivações, padrões de matrícula e papéis e responsabilidades únicos desses alunos adultos (KILGORE; RICE, 2003). Essa crescente população de alunos adultos vê a aprendizagem on-line como uma opção flexível e valiosa agora disponível para eles, pois equilibra o trabalho exigente, a família e outras responsabilidades.

Apesar da importância de se alçar conhecimento sobre os processos de admissão de estudantes que vem sendo adotados pelas instituições por meio de sistemas tradicionais, sistemas alternativos ou complementares ao vestibular, tais como: avaliação seriada (ou continuado) no ensino médio, o exame nacional do ensino médio (Enem) que processo usa o Sistema de Seleção Unificada (SISU), Programa Universidade para Todos (ProUni) e Fundo de Financiamento Estudantil (Fies) para viabilizar o acesso, vestibular agendado e prova eletrônica, e outras formas de acesso com a nota do Enem (Primeira fase do vestibular, Fase única, Parte da nota final e Bonificação) é de extrema relevância a identificação e compreensão dos fatores preditores anteriores aos processos seletivos de admissão na evasão voluntária de estudantes adultos neste estudo. Assim, o presente estudo tem como objetivo identificar e 
compreender os fatores preditores da evasão voluntária de estudantes adultos anteriores a admissão nas instituições de ensino superior a distância. Para tanto adota-se a pesquisa do tipo exploratória, pois o método envolve o levantamento de trabalhos de tese, dissertação e artigos científicos na literatura nacional e internacional. Esta pesquisa é parte de um estudo maior sobre os fatores preventivos da evasão de estudantes adultos, cuja temática abrange, a prevenção do abandono de estudantes no ensino superior a distância, evasão (dropout), e a sustentabilidade das IES no Brasil.

\section{Análise da literatura}

A análise de literatura oferece uma visão geral da pesquisa relacionada aos fatores anteriores a admissão como preditores de não-conclusão no ensino a distância neste estudo. Alguns estudos na literatura apontam fatores de forma (isolada ou em conjunto) presentes no indivíduo antes da sua admissão no Ensino Superior, que se associam com a permanência/evasão do estudante (BEAN, METZNER, 1985; PARKER, 1999; XENOS, PIERRAKEAS, PINTELAS, 2002; WALTER, 2006). Entre os diferentes fatores preditores que podem estar associados à evasão voluntaria de estudantes adultos no ensino superior a distância, encontram-se os anteriores a admissão. Nos diversos estudos sobre evasão de estudantes foram encontradas várias categorias de fatores preditores, tais como: experiências e variáveis de fundo, características demográficas, característica da clientela, características sociodemográfica do estudante, características individuais e características e habilidade do estudante, entre outras. Tais categorias de fatores dizem respeito ao conjunto de características e aspectos de caráter pessoal do estudante adulto não tradicional.

Buscando uma melhor forma de analisar seus estudos, Rovai (2003) estruturou seu modelo em quatro categorias de fatores, a saber: duas categorias de fatores prévios à admissão que são características dos estudantes e as suas habilidades, e duas categorias de fatores posteriores à admissão que são os fatores externos e os fatores internos.

Outros estudos analisam dados de cursos online (MORRIS, FINNEGAN, WU, 2005; LEVY, 2007; WOLFF, ZDRAHAL, NIKOLOV, PANTUCEK, 2013 ; VELHO, PINTO, 2015) apresentam pesquisas que utilizam dados de cursos já finalizados para compreender os padrões de comportamento e desempenho 
Educação superior a distância: fatores preditores da evasão anteriores a admissão de estudantes

dos estudantes adultos não tradicionais. Embora os estudos sejam importantes, estes ficam limitados em função de suas variáveis específicas, dificultando uma aplicação generalizada em qualquer contexto, além de olharem pelo retrovisor e não pelo farol.

Estudantes não tradicionais adultos tendem a diferir dos estudantes "tradicionais" de forma quantitativa e qualitativa. O termo "não tradicional" tem sido utilizado por outros pesquisadores para abranger uma ampla gama de características individuais que distinguem esses alunos com idade de 25 anos em diante da população geral que frequenta um programa de educação pós-secundária (STEWART, RUE, 1983; WEIDMAN, WHITE, 1985; BEAN, METZNER, 1985; FOX, 1986). De acordo com Rovai (2003), a definição do aluno não tradicional tem sido assunto de muita discussão na literatura profissional. Além disso, dentro e através do ensino superior, a definição de estudantes adultos varia (KE; XIE, 2009). Wyatt (2011) afirmou que existem dois grupos primários de estudantes que compõem a maioria das matrículas no ensino superior: Tradicional ( 18 a 24 anos) e não tradicional (25 ou mais). Segundo alguns autores, a maioria dos estudantes adultos de educação a distância são definidos entre as idades de 25 e 50 anos (MOORE, KEARSLEY, 2005; PARK, 2007; PARK, CHOI, 2009). Com base em uma revisão da literatura, Wladis, Hachey e Conway (2014) postularam que existe prova de que os alunos on-line são mais propensos a possuir características de estudante não tradicionais (ROVAI, 2003; PONTES, HASIT, PONTES, LEWIS, SIEFRING, 2010 ; WLADIS, HACHEY, CONWAY, 2014). Para a presente pesquisa de literatura dos estudos teóricos e empíricos da evasão, a ênfase maior do estudo está nos fatores preditores de estudantes adultos não tradicionais anteriores a admissão na modalidade de ensino a distância.

Embora o Modelo de integração do estudante Tinto (1975) seja um dos primeiros modelos a levantar a suposição de que os fatores característicos anteriores ao ingresso têm um efeito sobre o processo de evasão/permanência do estudante no Ensino Superior, este não foi desenvolvido para estudantes não tradicionais, tão pouco para IES a distância. No entanto o modelo de Tinto serviu de base para a maioria dos modelos teóricos que tentam explicar e prever a evasão de estudantes, a exemplo: o modelo não tradicional de atrito de estudantes Bean e Metzner (1985), Modelo de persistência composto de Rovai (2003) e Modelo teórico para o abandono de adultos na aprendizagem online de Park (2007). 
Ao propor o modelo não tradicional de atrito de estudantes Bean e Metzner (1985) definiram cinco fatores que poderiam prever a evasão: Experiencias e variáveis de fundo, variáveis acadêmicas, variáveis ambientais, variável de integração social e intenção de sair. Este modelo foi um dos primeiros a argumentar que fatores anteriores a admissão, como idade, gênero, status de registro, local de residência, os objetivos educacionais e a educação anterior podem estar direta ou indiretamente relacionados ao comportamento de evasão do estudante.

modelo de persistência composta de Rovai (2003) utilizado para analisar a evasão em cursos online incluiu duas categorias de fatores prévios à admissão que são características dos estudantes (idade, etnia, gênero, desenvolvimento intelectual, rendimento acadêmico) e as suas habilidades adquiridas em suas experiências de vida (letramento digital, letramento informacional, administração do tempo, leitura e escrita etc.) e duas categorias de fatores posteriores à admissão que fatores externos (situação econômica, jornada de trabalho, apoio familiar e crise de vida etc.) e os fatores internos (integração acadêmica, integração social, identidade com a escola e desempenho de pontuação etc.)

No Modelo teórico para o abandono de adultos na aprendizagem online (PARK, 2007) revisou estudos que enfocaram a identificação de fatores significativos que afetam estudantes de programas on-line não tradicionais e não graduados, que desistiram e propôs uma estrutura baseada no modelo de Composição da Persistência (ROVAl, 2003) para entender a evasão de adultos. $\bigcirc$ modelo proposto por Park (2007) apresenta os fenômenos evasão e permanência em cursos a distância diretamente influenciados por fatores internos e externos relacionados aos cursos e indiretamente influenciados por características e habilidades dos estudantes, sendo estas anteriores e independentes em relação ao curso. Park (2007) concentrou seu modelo em três principais categorias: características individuais (idade, gênero, escolaridade e situação de emprego), fatores externos e fatores internos.

Outros especialistas nacionais e internacionais, que se preocuparam em desenvolver estudos explicativos e preventivos da evasão, também apontam sobre a importância dos fatores anteriores a admissão para a compreensão dos motivos que levam a evasão dos estudantes. 
Educação superior a distância: fatores preditores da evasão anteriores a admissão de estudantes

Garrison (1987), por exemplo, na sua revisão para fins de compreensão e previsão da evasão escolar a distância foi um dos primeiros estudos focado em pesquisa de fatores demográficas.

Parker (1999) realizou um estudo sobre várias variáveis como preditores de abandono de alunos de cursos a distância. $\bigcirc$ foco de seu estudo foram os fatores lócus de controle e características demográficas como sexo, idade e número de horas empregadas como os principais preditores de abandono ou sucesso em cursos de e-learning.

Para Xenos, Pierrakeas e Pintelas (2002), a evasão (dropout), principal preocupação de instituições de ensino a distância, é causada por múltiplos fatores endógenos e exógenos ao curso. Há alguns resultados relevantes de pesquisas, citados por esses autores, indicando que um dos principais fatores que afetam a evasão é a quantidade de módulos completada pelo aluno. As pesquisas mostram, por exemplo, que, na maior parte dos casos, os estudantes que interromperam sua participação em um curso a distância o fizeram logo após o primeiro ou segundo módulo. Ainda segundo estes autores, a fatores que, historicamente, vêm afetando os níveis de evasão em cursos universitários a distância e que podem ser classificados em três grandes categorias: a) fatores internos relacionados às percepções do aluno e seu lócus de controle - interno-externo; b) fatores relativos ao curso e aos tutores; e, cl fatores relacionados a certas características demográficas dos estudantes, como idade, sexo, estado civil, número de filhos, tipo de trabalho ou profissão, entre outras. Os fatores mencionados em a e c correspondem a características da clientela, enquanto os fatores definidos em b se referem às características do treinamento.

Zerbini (2003) ressalta que, em cursos a distância, as variáveis referentes a características da clientela provavelmente assumiriam maior relevância na explicação de impacto do que em situações de treinamento presencial, dado que, em EaD, exige-se mais do participante com relação às competências de autocontrole, autoavaliação, administração do tempo e controle da ansiedade.

Nos estudos empíricos recentes Jun (2005) descobriu que o fator variáveis individuais de fundo, como o número de cursos on-line concluídos, sexo, horas de aprendizado do curso por semana e horas trabalhadas por semana e atenção, foram significativas para prever a evasão do aprendiz. 
Em grande parte dos estudos apresentados é demonstrado que as variáveis demográficas parecem ter um peso significativo na permanência/evasão do estudante. Segundo Yasmin (2013), a maioria dos pesquisadores do ensino a distância consideraram as variáveis demográficas dos alunos, como sexo, idade, número de filhos, status de emprego e região de residência, como os fatores que mais predizem o comportamento de abandono (WOODLEY; PARLETT, 1983; BELAWATI, 1998). Entretanto, entre essas variáveis, a idade (REKKEDAL, 1972; COOPER, 1990; XENOS; PIERRAKEAS; PINTELAS, 2002; NEWELL, 2007) e gênero (MARTIN, 1990) emergiram como os fatores mais capazes de prever as taxas de abandono e sucesso.

Ao contrário da maioria dos resultados de estudos dos especialista na previsão da evasão, o estudo de Willging e Johnson (2004) na Universidade de Illinois em Urbana-Champaign em um programa de pós-graduação online para investiga a existência de fatores que podem prever a probabilidade de um aluno evadir um programa on-line por meio do método de pesquisa eletrônica, mostram que as variáveis demográficas não preveem a probabilidade de sair de um programa. No entanto, eles observam que a única variável significativa identificada pela análise de regressão logística que poderia prever a probabilidade de abandono foi o grade point averages (GPA), mas a análise da tabela de classificação para essa variável indica que o GPA não é um forte preditor.

De modo geral, percebe-se que ainda são poucos os estudos que apresentam trabalhos com metodologia qualitativa, concretos para prever o resultado do estudante, que consideram as características individuais dos estudantes não tradicionais adultos anteriormente a admissão na graduação. Assim, os fatores anteriores a admissão de estudantes nas IES a distância em sua maioria se resume nas características do estudante ou denominações semeIhantes e habilidades do estudante. Uma revisão de pesquisa relacionada a esses fatores é necessária para atender o objetivo deste estudo e auxiliar as direções futuras dos estudos sobre prevenção da evasão de estudantes on-line.

\section{Objetivo da pesquisa}

As características de estudantes anteriores a admissão nas IES a distância têm sido objeto de pesquisa por muitos especialistas no assunto, que buscam prever e explicar a evasão de estudantes através de estudos com metodologias 
Educação superior a distância: fatores preditores da evasão anteriores a admissão de estudantes

qualitativa, quantitativa ou mista. Zerbini (2003; 2007) em seu estudo, propõe a investigação das características de cunho individual de estudantes por meio das variáveis antecedentes a evasão, de acordo com a pesquisadora, tais variáveis contribuem para a compreensão da evasão e da persistência em treinamentos a distância. Pesquisadores que adotam a metodologia quantitativa afirmaram que vários fatores demográficos de estudantes foram usados para prever ocorrências de desistências na literatura (INAN, YUKSELTURK, GRANT, 2009; LYKOURENTZOU, GIANNOUKOS, NIKOLOPOULOS, MPARDIS, LOUMOS, 2009). Os estudos que adotam a metodologia quantitativa analisam dados do comportamento e da interação do aluno no Ambiente Virtual de Aprendizagem (AVA) ao longo das atividades propostas nos cursos, com uso de técnicas de mineração de dados. $\bigcirc$ objetivo deste estudo é identificar e compreender os fatores preditores da evasão voluntária de estudantes adultos anteriores a admissão nas instituições de ensino superior a distância com base nos estudos com metodologia qualitativa. $\bigcirc$ quadro 1 apresenta autor e ano, Tipo de Estudo (TE), sendo (a) teórico (b) empírico, as categorias dos fatores preditivos anteriores a admissão e os principais fatores preditivos da evasão anteriores a admissão levantados na literatura e que têm servido como referên10 cias para o desenvolvimento de estudos e novos avanços.

\section{Quadro 1}

Principais fatores preditivos da evasão anteriores a admissão

\begin{tabular}{|c|c|c|c|}
\hline Autor (s) e Ano & TE & $\begin{array}{c}\text { Categorias de fatores } \\
\text { preditores anteriores } \\
\text { a admissão }\end{array}$ & Fatores preditores da evasão \\
\hline Bean e Metzner(1985) & a & Experiências e variáveis de fundo & $\begin{array}{l}\text { Idade, sexo, status de registro, local de } \\
\text { residência (região geográfica), objetivos } \\
\text { educacionais e a educação anterior }\end{array}$ \\
\hline Parker (1999) & b & Características demográficas & $\begin{array}{l}\text { Idade, sexo, número de horas empregadas e } \\
\text { número de cursos concluídos a distância }\end{array}$ \\
\hline \multirow{2}{*}{ Shin e Kim (1999) } & \multirow{2}{*}{$b$} & Características demográficas & (GPA) desempenho acadêmico \\
\hline & & Habilidades do estudante & Habilidades no gerenciamento de tempo \\
\hline \multirow{2}{*}{ Osborn (2001) } & \multirow{2}{*}{$b$} & Características demográficas & $\begin{array}{l}\text { GPA, nível de educaccão (ed. anterior) e número } \\
\text { de cursos anteriores de ensino a distância }\end{array}$ \\
\hline & & Habilidades do estudante & $\begin{array}{l}\text { Habilidades no gerenciamento de tempo } \\
\text { e Confiabilidade computacional }\end{array}$ \\
\hline
\end{tabular}




\section{Quadro 1}

Principais fatores preditivos da evasão anteriores a admissão (cont.)

\begin{tabular}{|c|c|c|c|}
\hline Autor (s) e Ano & TE & $\begin{array}{l}\text { Categorias de fatores } \\
\text { preditores anteriores } \\
\text { a admissão }\end{array}$ & Fatores preditores da evasão \\
\hline Cheung e Kan (2002) & $b$ & Características demográficas & $\begin{array}{l}\text { (GPA) desempenho acadêmico, número de cursos anteriores } \\
\text { concluídos online e experiência anterior relevante no campo }\end{array}$ \\
\hline \multirow{2}{*}{$\begin{array}{l}\text { Xenos, Pierrakeas e } \\
\text { Pintelas (2002) }\end{array}$} & \multirow[b]{2}{*}{$b$} & Características demográficas & $\begin{array}{l}\text { idade, sexo, estado civil, número de filhos, experiência } \\
\text { anterior relevante no campo e tipo de trabalho ou profissão }\end{array}$ \\
\hline & & Habilidades do estudante & $\begin{array}{l}\text { Subestimação do tempo necessário para equilibrar } \\
\text { suas obrigaç̦óes acadêmicas e profissionais } \\
\text { (Habilidades no gerenciamento de tempo) }\end{array}$ \\
\hline \multirow[b]{2}{*}{ Rovai (2003) } & \multirow[b]{2}{*}{ a } & Características dos estudantes & $\begin{array}{l}\text { idade, etnia, sexo, desenvolvimento intelectual } \\
\text { e (GPA)desempenho acadêmico }\end{array}$ \\
\hline & & Habilidades do estudante & $\begin{array}{l}\text { alfabetização em informática, alfabetização de } \\
\text { informaç̃oes, gerenciamento de tempo, habilidades de } \\
\text { leitura e escrita, e habilidades de interação on-line }\end{array}$ \\
\hline \multirow{2}{*}{ Dupin-Bryant (2004) } & \multirow{2}{*}{$b$} & Variáveis de fundo e Experiências & $\begin{array}{l}\text { GPA, nivel de educacãa (ed. anterior) e número } \\
\text { de cursos anteriores concluídos online }\end{array}$ \\
\hline & & Habilidades do estudante & $\begin{array}{l}\text { Treinamento de informática anterior relevante } \\
\text { (alfabetização em informática) }\end{array}$ \\
\hline $\begin{array}{l}\text { Willging e Johnson } \\
\text { (2004) }\end{array}$ & $b$ & Características demográficas & GPA (desempenho acadêmico) \\
\hline $\begin{array}{l}\text { Abbad, Carvalho e } \\
\text { Zerbini (2006) }\end{array}$ & $b$ & Característica da Clientela & $\begin{array}{l}\text { Idade, sexo, região geográfica e nível } \\
\text { de escolaridade (ed. anterior) }\end{array}$ \\
\hline Walter (2006) & $b$ & Características individuais & $\begin{array}{l}\text { idade, gênero, participação anterior em curso } \\
\text { a distância, pagamento do curso pelo aluno } \\
\text { e valor instrumental do treinamento }\end{array}$ \\
\hline Newell (2007) & $b$ & Características individuais & Idade e etnia \\
\hline Park (2007) & a & Características individuais & $\begin{array}{l}\text { idade, gênero, escolaridade (ed. } \\
\text { anterior) e situação de emprego }\end{array}$ \\
\hline Aragon (2008) & $b$ & Características individuais & GPA (desempenho acadêmico) \\
\hline $\begin{array}{l}\text { Walter e Abbad } \\
\qquad(2008)\end{array}$ & $b$ & Característica da Clientela & $\begin{array}{l}\text { idade, sexo, participação anterior em curso a } \\
\text { distância e pagamento do curso pelo aluno }\end{array}$ \\
\hline Umekawa (2014) & $b$ & $\begin{array}{l}\text { Características sociodemográfica } \\
\text { do estudante }\end{array}$ & idade, sexo, estado civil e composição familiar \\
\hline
\end{tabular}

Fonte: Elaborado pelos autores. 
Educação superior a distância: fatores preditores da evasão anteriores a admissão de estudantes

Embora alguns estudiosos tenham centrado seus estudos de evasão de estudantes adultos no programa sobre variáveis idade, sexo e nível de educação (BEAN, METZNER, 1985; ROVAI, 2003; DUPIN-BRYANT, 2004; ABBAD, CARVALHO, ZERBINI, 2006; PARK, 2007). Outros estudos centraram suas atenções nas variáveis estado civil, participação anterior em curso a distância, GPA e Habilidades no gerenciamento de tempo (XENOS, PIERRAKEAS, PINTELAS, 2002; ROVAI, 2003; DUPIN-BRYANT, 2004; WALTER, 2006; HOLDER, 2007; WALTER, ABBAD, 2008; UMEKAWA, 2014). A revisão de literatura com foco nos fatores anteriores a admissão, especificamente das características individuais de estudantes, identificou vários fatores preditores da evasão anteriores a admissão de estudantes adultos, tornando evidente que este tema tem recebido crescente atenção de pesquisadores que buscam compreender e prever a evasão de estudantes adultos nas IES a distância. A exceção dos estudos de Shin e Kim (1 999), Osborn (2001), Xenos, Pierrakeas e Pintelas (2002), Rovai (2003) e Dupin-Bryant (2004) que possuem duas categorias de fatores anteriores a admissão de estudantes (características dos estudantes e habilidades) todos os demais especialistas neste estudo tem uma única categoria de fator preditor anterior a admissão, apesar das várias denominações das

12 características individuais do estudante, a saber: experiências e variáveis de fundo, características demográficas, característica da clientela, características individuais e características e habilidade do estudante. Características individuais dos estudantes no momento da entrada na graduação são definidas como os fatores de pré-requisito para a prevenção da evasão de estudantes em todos os estudos acima mencionados. Os estudos mencionados no quadro 1 fizeram contribuições significativas no campo da previsão do abandono na modalidade de ensino a distância ao destacarem as características individuais dos estudantes adultos. Normalmente, essas características individuais são possíveis quando coletadas durante o registro de entrada dos estudantes pela maioria das instituições, que formam um banco de informações e são acessadas e usadas pelos gestores em tempo real por meio dos sistemas de informação das instituições educacionais. Assim, os conjuntos de atribuições das características individuais dos estudantes que compõem este estudo permitiram identificar os principais fatores preditores da evasão de estudantes. 


\section{Metodologia da pesquisa}

Esta pesquisa é do tipo exploratória, pois o método envolveu o uso de recursos de biblioteca on-line para o levantamento de trabalhos de teses, dissertações e artigos científicos na literatura nacional e internacional. As bases de dados pesquisadas incluíram o seguinte: Google Scholar, Education Resources Information Center (ERIC) e EBSCO HOST. Os termos-chave que se encaixam nos domínios deste estudo foram utilizados para definir o fundamento desta revisão de literatura: foram empregadas todas as combinações e permutações de abandono, attrition, dropout, withdrawal, admissão, educação à distância, evasão, fatores, fator e predicto. A pesquisa de literatura sobre os fatores preditores da evasão anteriores a admissão de estudantes nas IES a distância tomou por base, a partir dos estudos do modelo não tradicional de atrito de estudantes Bean e Metzner (1985). Esse estudo foi um dos primeiros a argumentar que fatores anteriores a admissão, como idade, gênero, status de registro, local de residência, os objetivos educacionais e a educação anterior podem estar diretos ou indiretamente relacionados ao comportamento de evasão. Isso direcionou o ponto focal deste estudo para a construção dos resultados da pesquisa.

\section{Resultados da pesquisa}

Com base na avaliação da metodologia qualitativa dos estudos teóricos e empíricos coletados na literatura consultada, e considerando o foco do objetivo deste estudo nas características individuais dos estudantes a partir dos resultados do quadro 1, 25 fatores preditores da evasão foram saturados dos 66 fatores identificados. Classificamos esses 25 fatores em duas categorias principais: a) Características dos estudantes; e, b) Habilidades prévias dos estudantes.

Várias observações podem ser feitas sobre os fatores preditores da evasão anteriores a admissão de estudantes contidos a partir do gráfico 1 . Primeiro, com base em estudos anteriores, pode-se classificar duas categorias de fatores (características dos estudantes e habilidades previas dos estudantes) que de forma combinada ou não podem contribuir para o desenvolvimento de políticas da instituição, que tenham por finalidade a prevenção da evasão. 
A primeira categoria, característica do estudante consiste nas características do processo educacional no momento da entrada, como preparação educacional, stałus socioeconômico, situação sócio demográfico e experiencias anteriores. Essas características são fixas ou mudam lentamente durante a duração do envolvimento de um estudante com uma instituição de ensino à distância e, como tal, exercem uma influência relativamente constante nas chances de sucesso dos alunos. A segunda categoria, habilidades prévias, consiste basicamente nas habilidades com tecnológicas necessárias para que - estudante possa desenvolver suas atividades da graduação e habilidades de gerenciamento de tempo, que incluí a capacidade de estimar o tempo e o esforço necessários para realizar as tarefas relacionadas a sua graduação.

\section{Gráfico 1}

Frequência relativa com a qual vários fatores preditores da evasão anteriores a admissão de estudantes foi mencionada em estudos anteriores

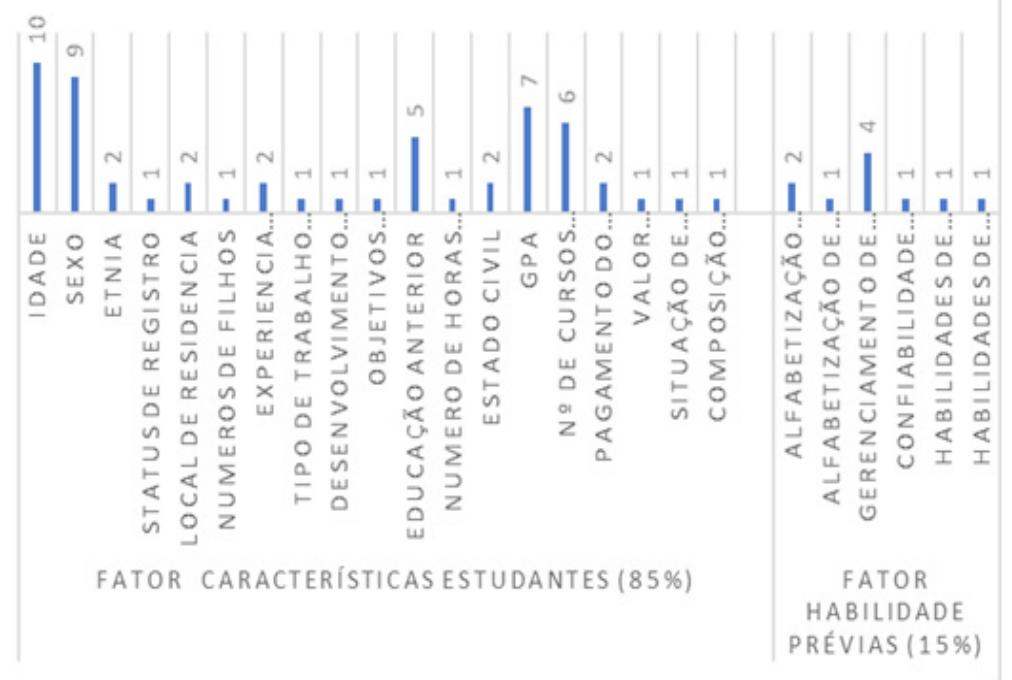

Fonte: Elaborado pelos autores.

Em segundo lugar, embora esses fatores sejam de extrema importância e úteis para os avanços dos estudos no campo da prevenção da evasão, faz-se necessário uma maior compreensão das combinações entre esses fatores para torná-los ainda mais significativas na prevenção da evasão de estudantes. 
Por fim, sintetizamos esses fatores tomando como base os estudos teóricos do modelo não tradicional de atrito de estudantes de Bean e Metzner (1985), do modelo de persistência composta da Rovai (2003) e do Modelo teórico para o abandono de adultos na aprendizagem online (PARK, 2007), pois estes modelos abordam uma variedade de fatores relacionados aos aspectos das características individuais dos estudantes adultos e não tradicionais.

No ambiente de aprendizado na modalidade EAD, a capacidade do estudante adulto de trabalhar, manter o foco nos objetivos pessoais e acadêmicos, e demonstrar habilidades acadêmicas gerais e habilidades técnicas relevantes está entre algumas das qualidades e abordagens de vida que aumentam o sucesso de conclusão da graduação. Assim, no gráfico 1, descrevemos os resultados da compreensão das duas principais categorias de fatores e seus respectivos fatores anteriores a admissão de estudantes.

\section{Categoria 1- Característica dos estudantes}

A categoria de fatores características dos estudantes foram os fatores mais citados nos estudos revisados, ocupando aproximadamente $85 \%$ do número total de fatores preditores da evasão identificados 156 de 66 fatores, - gráfico 1). Apresentamos uma síntese dos dados relevantes para a abordagem das ações institucionais, visando à prevenção da evasão de estudantes. Os fatores Características dos estudantes vem sendo muito citado pelos autores para prever e explicar o fato de alunos desistirem ou persistirem nos cursos e é quase um consenso a sua influência sobre o fenômeno evasão. Dividimos essa categoria de fatores preditores da evasão do estudante em 19 subcategorias, incluindo "idade"' (15.3\%, 10 fatores), "sexo"' (13.7, 9 fatores), "etnia"' (3\%, 2 fatores), "status de registro"' (1.5\%, 1 fator), "local de residência"' $13 \%, 2$ fatores), "número de filhos"' (1.5\%, 1 fator), "experiência anterior relevante no campo" (3.0\%, 2 fatores), "tipo de trabalho ou profissão"' (1.5\%, 1 fator), "desenvolvimento intelectual"' (1.5\%, 1 fator), "objetivos educacionais" (1.5\%, 1 fator), "educação anterior" (7.7\%, 5 fatores), "número de horas empregadas" (1.5\%, 1 fator), "estado civil" (3\%, 2 fatores), "GPA" (10.6\%, 7 fatores), "número de cursos concluídos a distância" (9.2\%, 6 fatores), "pagamento do curso pelo aluno" (3\%, 2 fatores), "valor instrumental do treinamento" $11.5 \%, 1$ fator), "situação de emprego" (1.5\%, 1 fator) e "composição familiar" (1.5\%, 1 fator). 
Esta categoria refere-se à situação sócio demográfica e experiências anteriores, que correspondem às características dos estudantes para a prevenção da evasão neste estudo. São denominados fatores características dos estudantes: idade, sexo, etnia, status de registro, local de residência, os objetivos educacionais, educação anterior, número de horas empregadas, estado civil, GPA, número de cursos concluídos a distância, pagamento do curso pelo aluno, valor instrumental do treinamento, situação de emprego e composição familiar. De acordo com Zerbini e Abbad (2010), as características dos estudantes podem exercer uma interferência mais significativa nos resultados de ações educacionais a distância do que naqueles de natureza presencial, uma vez que a aprendizagem efetiva está, nesse primeiro caso, subordinada muito mais ao empenho empreendido pelo próprio sujeito lautogerenciamento da aprendizagem) do que aos recursos educacionais disponíveis. Alinhado nesse mesmo entendimento Lee e Choi (2011) afirma que:

Alunos que tiveram experiências anteriores relacionadas ao conteúdo de um curso, ou que frequentaram cursos superiores, tinham maior probabilidade de concluir o curso. Independentemente da qualidade do desempenho dos alunos em tais experiências, os alunos bem-sucedidos e os alunos que abandonaram os cursos de e-learning diferiram significativamente em relação à quantidade de experiência acadêmica e profissional que tinham antes de fazer cursos on-line. Levy (2007) revelou que os alunos com maior nível de escolaridade e / ou mais anos de escolaridade eram menos propensos a abandonar os cursos do que seus pares. Outros pesquisadores também descobriram que o número de cursos on-line anteriores concluídos foi um importante preditor de abandono (Cheung e Kan, 2002; Dupin-Bryant, 2004; Osborn, 2001). Xenos, Pierrakeas e Pintelas (2002) examinaram a experiência acadêmica e profissional anterior de 1.230 alunos em cursos de informática. Os estudantes que haviam completado cursos prévios no campo da Informática ou estavam envolvidos em programação profissional ou atividades de processamento de dados tiveram taxas de evasão significativamente menores do que os estudantes sem tais experiências [...] (CHEUNG; KAN, 2002, p. 607, tradução nossa).

Outros estudos que abordam a relação entre gênero e evasão encontram-se aqueles que apontam para os índices de evasão entre homens e mulheres, buscando conhecer diferenças na evasão para algum dos sexos; 
e aqueles que verificam quais são os determinantes mais significativos para a evasão dos estudantes do sexo masculino e do sexo feminino, buscando conhecer os aspectos que podem estar associados à permanência ou evasão dos estudantes.

No estudo empírico de Xenos, Pierrakeas e Pintelas (2002) - realizado em uma amostra de estudantes de um curso de graduação em Informática a distância - avaliou o rendimento e a evasão de alunos. Evasão, nesse estudo, foi definida como o percentual de alunos que se matricularam no curso, mas nunca o iniciaram, ou que o começaram, mas interromperam sua participação. Os principais resultados mostram que maiores índices de evasão ocorreram entre os alunos mais idosos, com idade igual ou superior a 35 anos; entre os homens, que mostraram maiores índices de evasão que as mulheres; entre os alunos que estudaram menos destacando o importante papel de atividades presenciais na manutenção de alunos em cursos a distância. Umekawa (20 14) realizou uma pesquisa com o objetivo de propor e testar um modelo de avaliação de ações educacionais ofertadas a distância, buscando identificar fatores preditores de elemento ligados a evasão e persistencia academica (contexto de estudo, desenho do treinamento e o proprio estudante) relacionado as caracteristica da clientela (dados socios demograficos e estrategias de aprendizagem). Os resultados de seus estudos apontam que os fatores estado civil e composição familiar e as estrategias de aprendizagem explicam os fatores ligados a evasão e a permanência no EAD. Outros estudos investigaram a relação entre estado civil e resultado acadêmico. Powell, Conway e Ross ( 1990 apud ROSS; POWELL, 1990) descobriram que o estado civil estava relacionado ao desempenho acadêmico, indicando que os estudantes aumentaram suas chances de sucesso por terem cônjuges ou parceiros de apoio. Chinnanon (1985) também descobriu que o estado civil tem um efeito significativo na realização educacional, particularmente em cursos de experiência de vida. Os estudos de Shin e Kim (1999), Osborn (2001), Cheung e Kan (2002), Rovai (2003), Dupin-Bryant (2004), Willging e Johnson (2004) e Aragon e Johnson (2008) afirmam que as características individuais preveem a evasão e indicam que os alunos com GPAs mais baixos correm maior risco de abandonar seus cursos on-line. 
Educação superior a distância: fatores preditores da evasão anteriores a admissão de estudantes

\section{Categoria 2 - Habilidades prévias dos estudantes}

Além da categoria 1 - Característica dos estudantes em áreas relevantes para um curso na modalidade de ensino a distância, a categoria 2- habilidades prévias dos estudantes também foi identificada como uma característica individual do estudante que pode ter possível correlação com sua decisão de desistir. Essa categoria ocupou $15 \%$ do número total de fatores preditores da evasão identificados (10 de 66 fatores, gráfico 1). Dividimos essa categoria de fatores preditores da evasão do estudante em seis subcategorias, incluindo "alfabetização de informática" (3\%, 2 fatores), "alfabetização de informações" (1.5\%, 1 fator), "gerenciamento de tempo" (6\%, 4 fatores), "confiabilidade computacional" (1.5\%, 1 fator), "habilidades de leitura e escrita" (1.5\%, 1 fatores) e "habilidades de interação on-line" (1.5\%, 1 fator).

Assim, se nota que os fatores identificados no estudo lidaram principalmente com duas habilidades principais: habilidades com tecnológicas e habilidades de gerenciamento de tempo. De acordo com (DEIMANN, BASTIAENS, 2010; ROMERO, BARBERÀ, 20111 l a falta de habilidades do estudante de fazer uso das ferramentas eletrônicas disponibilizadas e a inabili-

18 dade de organizar o tempo de estudo influenciam a evasão do estudante. Já o estudo de (MOORE; SENER; FETZNER, 2006) sugere que os alunos que prosperam em um ambiente de aprendizado na modalidade EAD parecem possuir qualidades e abordagens da vida, além de serem academicamente preparados, incluindo atributos como auto eficácia do computador e habilidades de gerenciamento de tempo. Pesquisas mais recente indicam que indivíduos que experimentam maiores dificuldades em relação ao manejo dos instrumentais eletrônicos do curso possuem maiores chances de evadirem-se dos programas educacionais (ALMEIDA, 2007; SILVA, TOMAZ, 2006). As habilidades tecnológicas incluem conhecimentos de informática relevantes para o formato de entrega ou conteúdo de cursos on-line, como pesquisa na Internet, gerenciamento de arquivos, aplicativos de Internet e sistemas operacionais de computador (OSBORN, 2001; ROVAI, 2003; DUPIN-BRYANT, 2004). Para lidar com as habilidades de gerenciamento de tempo incluíram a capacidade de estimar o tempo e o esforço necessários para realizar uma tarefa (SHIN; KIM, 1999; OSBORN, 2001 ; XENOS, PIERRAKEAS, PINTELAS, 2002; ROVAI, 20031. Pesquisas sobre essas habilidades indicaram que elas eram preditores significativos na previsão da evasão em cursos on-line. Diversas pesquisas 
e frequência de uso dos recursos e instrumentos tecnológicos nos Ambientes Virtuais de Aprendizagem (AVAs) podem influenciar a decisão de evasão ou não do estudante (ABBAD, CARVALHO, ZERBINI, 2006; ALMEIDA, 2007; PALLOFF, PRATT, 2004; VARGAS, 2004; XENOS, PIERRAKEAS, PINTELAS, 2002). Para Silva e Tomaz (2006), possivelmente a falta de contato prévio e de habilidade com o uso das estruturas tecnológicas dos cursos são um dos principais fatores que influenciam os participantes a desistirem do mesmo.

\section{Discussões, contribuições, limitações e pesquisas futuras}

Discussões das descobertas

A partir da literatura consultada, quatro distinções do status da pesquisa sobre os fatores preditores da evasão anteriores a admissão de estudantes na educação a distância foi evidente. Primeiro, ainda são poucos os estudos teóricos que tratam do tema prevenção da evasão na educação a distância. Em segundo, apesar dos vários estudos empíricos encontrados, as pesquisas parecem estar indo em muitas direções diferentes simultaneamente, ao mesmo tempo em que produz resultados contraditórios. Em terceiro, os estudos com abordagem qualitativa descritos nesta pesquisa, incluem os fatores preditores de evasão na educação a distância que os autores afirmavam serem os mais proeminentes. Em quarto, Duas categorias de fatores preditores da evasão anteriores a admissão de estudantes na educação a distância foram identificadas, incluindo a Categoria 1 - fatores características dos estudantes, sendo essa a mais citadas nos estudos revisados, ocupando aproximadamente 85\% do número total de fatores preditores da evasão identificados e Categoria 2- habilidades prévias dos estudantes também foi identificada como uma característica individual do estudante que pode ter possível correlação com sua decisão de desistir. Essa categoria ocupou 15\% do número total de fatores preditores da evasão identificados. Assim, a Categoria 1 - fatores características dos estudantes evidencia a direção dos estudos de fatores preditores da evasão anteriores a admissão de estudantes na educação a distância. 
Educação superior a distância: fatores preditores da evasão anteriores a admissão de estudantes

\section{Contribuições do estudo}

Embora a pesquisa disponível ofereça vários fatores preditores da evasão anteriores a admissão de estudantes na educação a distância, há pouca oferta de fatores que pode melhorar prontamente a situação da evasão na educação a distância de forma isolada. Apesar dessa oferta, os estudos revisados na pesquisa indicam que os fatores "idade, sexo e educação anterior", presentes nos estudos de Bean e Metzner (1985), Parker (1999), Xenos, Pierrakeas e Pintelas (2002), Rovai (2003), Abbad, Carvalho e Zerbini (2006), Walter (2006) e Walter e Abbad (2008), evidenciam a importância desses fatores na prevenção da evasão de estudantes nas IES a distância. $\bigcirc$ fator "GPA" presente nos estudos de Shin e Kim (1999), Osborn (2001), Cheung e Kan (2002), Rovai (2003), Dupin-Bryant (2004), Willging e Johnson (2004) e Aragon e Johnson (2008) afirmam que as características individuais preveem a evasão e indicam que os alunos com GPAs mais baixos correm maior risco de abandonar seus cursos on-line. $\bigcirc$ fator "número de cursos a distância concluídos" presente nos estudos de Parker (1 999), Osborn (2001), Cheung e Kan (2002), Walter (2006) e Walter e Abbad (2008) indica que, quanto mais

20 cursos concluídos em programas de educação a distância menor é a probabilidade de evasão. $\bigcirc$ fator habilidades de gerenciamento de tempo presente nos estudos de Shin e Kim (1 999), Osborn (2001), Xenos, Pierrakeas e Pintelas (2002) e Rovai (2003) evidenciam o equilíbrio do tempo que estudante deve ter na realização das tarefas referentes a sua graduação e outras atividades ou compromissos. Assim, os fatores idade, sexo e educação anterior, GPA, número de cursos concluídos a distância e habilidades de gerenciamento de tempo indicaram ser preditores significativos na previsão da evasão em cursos na educação a distância.

\section{Limitações}

Embora os estudos teóricos e empíricos anteriores à admissão da graduação do estudante sejam interessantes, a limitação consiste em apresentar fatores muito específicos, os quais podem ser aplicados em qualquer contexto. Percebe-se que os poucos estudos teóricos, apresentam modelos concretos que explicam ou preveem o resultado do aluno, considerando seu perfil anterior a admissão na graduação. Escolhemos uma abordagem qualitativa em função 
de não haver preocupação com uma representatividade numérica, mas, sim, com a identificação e aprofundamento da compreensão dos fatores. Assim, este estudou ficou limitado quanto a abordagem quantitativa.

\section{Sugestões para pesquisas futura}

Apesar da importância dos fatores preditores da evasão anteriores a admissão de estudantes na educação a distância para prevenção da evasão, pesquisas futuras são necessárias não apenas para verificar o que descrevemos, mas que possam sistematiza os conhecimentos de forma isolada ou em conjunto com outros fatores para o aperfeiçoamento de instrumentos de apoio a prevenção da evasão. Pesquisa futuras devem também ter uma abordagem quantitativa para complementar e ampliar nossos estudos.

\section{Nota}

1 Cf. Prensky (2001) considera os nativos digitais todos os que nasceram rodeados e estão imerso no mundo digital, repleto de tecnologias como computadores, games, mp3, entre outros. Já os imigrantes digitais são aqueles que estão aprendendo e se adaptando ao uso de "novas" tecnologias.

\section{Referências}

ABBAD, Gardênia; CARVALHO, Renata Silveira; ZERBINI, Thaís. Evasão em curso via internet: explorando variáveis explicativas. Revista de Administração de Empresas Eletrônica, São Paulo, v. 5, n. 2, p. 1-26, 2006. Disponível em: <http://www.scielo.br/pdf/raeel/ v5n2/v5n2a08.pdf>. Acesso em: 10 set. 2017.

ALMEIDA, Onília Cristina. Evasão em cursos a distância: validação de instrumento, fatores influenciadores e cronologia da desistência. 2007. 177f. Dissertação - Programa de PósGraduação em Administração, Universidade de Brasília, 2007. Disponível em: <http:// bdtd.bce.unb.br/tedesimplificado/tde_arquivos/65/TDE-2007-10- 26T170707Z-1970/ Publico/Dissert_Onilia\%20Cristina.pdf>. Acesso em: 10 set. 2017.

ARAGON, Steven; JOHNSON, Elaine. Factors influencing completion and non-completion of community college online courses. American Journal of Distance Education, Filadélfia, v. 22, n. 3, p. 146-158, 2008. 
Educação superior a distância: fatores preditores da evasão anteriores a admissão de estudantes

ASSOCIAÇÃO BRASILEIRA DE EDUCAÇÃO À DISTÂNCIA. Censo EAD Brasil 2016: relatório analítico da aprendizagem a distância no Brasil. Curitiba: InterSaberes, 2017. Disponível em: <http://abed.org.br/censoead2016/Censo_EAD_2016_portugues.pdf>. Acesso em: 9 mar. 2018.

BEAN, John; METZNER, Barbara. A conceptual model of nontraditional undergraduate student attrition. Review of Educational Research, Thousand Oaks, v. 55, n. 4, p. 485-540, 1985.

BELAWATI, Tian. Increasing student persistence in Indonesian post-secondary distance education. Distance Education, Melbourne, v. 19, n. 1, p. 81-109, 1998.

BRASIL. Ministério da Educação. Instituto Nacional de Estudos e Pesquisas Educacionais Anísio Teixeira. Sinopse Estatística da Educação Superior 2016. Brasília: Inep, 2017. Disponível em: <http://portal.inep.gov.br/basica-censo-escolar-sinopse-sinopse>. Acesso em: 7 jun. 2018.

CHEUNG, Lenis L. W.; KAN, Andy C. N. Evaluation of factors related to student performance in a distance-learning business communication course. Journal of Education for Business, Abingdon, v. 77, n. 5, p. 257-263, 2002.

22 CHINNANON, Sanong. Multi-media distance education: a study of factors affecting the educational achievement of adult participants in the radio correspondence project in Thailand. Dissertation Abstracts International: a Humanities and Social Sciences. Ann Arbor, v. 46, n. 4, p. 870-871, 1985.

COOPER, Elizabeth. An analysis of student retention at Snead State Junior College Washibgton, DC: ERIC Clearinghouse, 1990.

DEIMANN, Marcus; BASTIAENS, Theo. The role of volition in distance education: An exploration of its capacities. International Review of Research in Open and Distance Learning, Athabasca, v. 11, n. 1, p. 1-16, 2010.

DUPIN-BRYANT, Palmela Annette. Pre-entry variables related to retention in online distance education. The American Journal of Distance Education, Filadélfia, v. 18, n. 4, p. 199206, 2004

FOX, Richard. Application of a conceptual model of college withdrawal to disadvantaged smdents. American Educational Research Journal, Thousand Oaks, v. 23, n. 3, p. 415424, 1986 
GARRISON, D. Randy. Researching dropout in distance education. Distance Education, Melbourne, v. 8, n. 1, p. 95-101, 1987.

GILLIS, Carol. Leaving seats empty: exploring student attrition in an Undergraduate Health Sciences Program. 2007. 104f. Thesis (Master of Arts in Education) - Mount Saint Vincent University, Halifax, 2007.

HOLDER, Burce. An investigation of hope, academics, environment, and motivation as predictors of persistence in higher education online programs. The Internet and Higher Education, Maryland Heights, v. 10, n. 4, p. 245-260, 2007.

INAN, Fethi; YUKSELTURK, Erman; GRANT, Michael. Profiling potential dropout students by individual characteristics in an online certificate program. International Journal of Instructional Media, Darien, v. 36, n. 2, p. 163-176, 2009.

JUN, Jusung. Understanding dropout of adult learners in e-learning. 2005. 146f. Thesis (Doctoral) - The University of Georgia, Georgia, 2005.

KE, Fengfeng; XIE, Kui. Toward deep learning for adult students in online courses. The Internet and Higher Education, Maryland Heights, v. 12, n. 3-4, p. 136-145, 2009.

KILGORE, Deborah; RICE, Penny. Meeting the special needs of adult students. Hoboken: Wiley Periodicals, 2003.

LEE, Youngju; $\mathrm{CHOI}$, Jaecho. A review of online course dropout research: Implications for practice and future research. Educational Technology Research \& Development, New York, v. 59, n. 5, p. 593-618, 2011 .

LEVY, Yair. Comparing dropouts and persistence in e-learning courses. Computers and Education, Amesterdã, v. 48, n. 2, p. 185-204, 2007.

LYKOURENTZOU, loanna; GIANNOUKOS, loannis; NIKOLOPOULOS, Vassilis; MPARDIS, George; LOUMOS, Vassili. Dropout prediction in e-learning courses through the combination of machine learning techniques. Computers and Education, Amesterdã, v. 53, n. 3, p. 950965, 2009.

MARTIN, Larry. Dropout, persistence and completion in adult second and pre-vocational education programs. Adult Literacy and Basic Education, Killarney, v. 14, n. 3, p. 159-174, 1990.

MONEREO, Carles; POZO, Juan Ignácio. $\bigcirc$ aluno em ambientes virtuais: condições, perfil e competências. In: COLL, Cesar; MONEREO, Carles. Psicologia da educação virtual: 
Educação superior a distância: fatores preditores da evasão anteriores a admissão de estudantes

aprender e ensinar com as tecnologias da informação e comunicação. Porto Alegre: Artmed, 2010. p. 136-145.

MOORE, Janet; SENER, John; FETZNER, Marie. Getting better: ALN and student success. Journal of Asynchronous Learning Networks, Newburyport, v. 10, n. 3, p. 55-84, 2006.

MOORE, Michael; KEARSLEY, Greg. Distance education: a systems view. 2nd ed. Belmont: Wadsworth Publishing, 2005.

MORRIS, Libbby; FINNEGAN, Catherine; WU, Sz-Shyan. Tracking student behavior, persistence, and achievement in online courses. The Internet and Higher Education, Maryland Heights, v. 8, n. 3, p. 221-231, 2005.

NEWELL, Chandler. Learner characteristics as predictors of online course completion among nontraditional technical college students. 2007. $113 \mathrm{f}$. Thesis (Doctor of Education) - The University of Georgia, Georgia, 2007.

OSBORN, Viola. Identifying at-risk students in videoconferencing and web-based distance education. American Journal of Distance Education, Filadélfia, v. 15, n. 1, p. 41-54, 2001.

PALLOFF, Rena; PRATT, Keith. O aluno virtual: um guia para trabalhar com estudantes on-line. Tradução Vinicius Figueira. Porto Alegre: Artmed, 2004.

PARK, Ji-Hye. Factors related to learner dropout in online learning. In: ACADEMY OF HUMAN RESOURCE DEVELOPMENT ANNUAL CONFERENCE, 2007. Indianapolis. Proceedings..., Indianapolis, 2007.

PARK, Ji-Hye; CHOl, Hee Jun. Factors influencing adult learners' decision to drop out or persist in online learning. Educational Technology \& Society, Taiwan, v. 12, n. 4, p. 207-217, 2009

PARKER, Angie. A study of variables that predict dropout from distance education. International Journal of Educational Technology, Washington, DC, v. 1, n. 2, p. 1-10, 1999.

PONTES, Manuel; HASIT, Cindi; PONTES, Nancy; LEWIS, Phillip; SIEFRING, Karen. Variables related to undergraduate students preference for distance education classes. Online Journal of Distance Learning Administration, Jekyll Island, v. 13, n. 2, 2010.

PRENSKY, Marc. Digital natives, digital immigrants. NBC University Press, Berkeley, v. 9, n. 5, p. 1-6, Oct. 2001. 
REKKEDAL, Torstein. Correspondence studies: recruitment, achievement, and discontinuation. Epistolodidaktika, Norway, v. 2, n. 3, p. 38, 1972.

ROMERO, Margarida; BARBERÀ, Elena. Quality of learners' time and learning performance beyond quantitative time-on-task. The International Review of Research in Open and Distance Learning, Athabasca, v. 12, n. 5, p. 125-137, 2011.

ROSS, Lynda; POWELL, Richard. Relationships between gender and success in distance education courses: a preliminary investigation. Research in Distance Education, Geelong, v. 2, n. 2, p. 10-11, 1990.

ROVAl, Alfred. In search of higher persistence rates in distance education online programs. The Internet and Higher Education, Maryland Heights, v. 6, n. 1, p. 1-16, 2003.

SHIN, Namin; KIM, Juhu. An exploration of learner progress and drop-out in Korea National Open University. Distance Education, Melbourne, v. 20, n. 1, p. 81 1-95, 1999.

SILVA, Deusimar Ribeiro; TOMAZ, José Batista. Por que a evasão? Ceará: Escola de Saúde Pública do Ceará, 2006.

STEWART, Sylvia RUE, Pennny. Commuter students: definition and distribution. In: STEWART, Sylvia (Ed.). Commuter students: enhancing their educational experiences. San Francisco: Jossey-Bass, 1983. p. 3-8.

TINTO, Vicent. Dropout from higher education: a theoretical synthesis of recent research. Review of Educational Research, Thousand Oaks, v. 45, n. 1, p. 89-125, 1975.

UMEKAWA, Elienay Eiko Rodrigues. Preditores de fatores relacionados à evasão e à persistência discente em ações educacionais a distância. 2014. 256f. Dissertação - Faculdade de Filosofia, Ciências e Letras, Universidade de São Paulo, São Paulo, 2014.

VARGAS, Miramar Ramos Maia. Barreiras à implantação de programas de educação e treinamento a distância. 2004. 185f. Tese - Instituto de Psicologia, Universidade de Brasilia, Brasilia, 2004.

VELHO, Deividi; PINTO, Andrio. As competências EaD de alunos concluintes do Ensino Médio: resultados do instrumento de coleta de dados. Revista Novas Tecnologias na Educação, Porto Alegre, v. 13, n. 1, p. 1-10, 2015.

WALTER, Amanda; ABBAD, Gardênia. Variáveis preditoras de evasão em dois cursos a distância. In: ENCONTRO ANUAL DA ASSOCIAÇÃO NACIONAL DE PROGRAMAS DE PÓS-GRADUAÇÃO EM ADMINISTRAÇÃO E PESQUISA, 32., 2008, Rio de janeiro. Anais... Rio de Janeiro: Anpad, 2008. 
Educação superior a distância: fatores preditores da evasão anteriores a admissão de estudantes

WALTER, Amanda Moura. Variáveis preditoras de evasão em cursos a distância. 2006.

177f. Dissertação - Instituto de Psicologia, Universidade de Brasília, Brasília, 2006.

WEIDMAN, John; WHITE, Richard. Postsecondai y "high-tech" training for women on welfare: correlates of program completior. Journal of Higher Education, Abingdon, v. 56, n. 5, p. 555-568, 1985.

WILLGING, Pedro; JOHNSON, Scott. Factors that influence students' decision to dropout of online courses. Journal of Asynchronous Learning Network, Newburyport, v. 8, n. 4, p. 105-1 18, 2004.

WLADIS, Claire; HACHEY, Alyse; CONWAY, Kachey. The representation of minority, female, and non-traditional STEM majors in the online environment at community colleges: a nationally representative study. Community College Review, North Carolina, v. 43, n. 1, p. 89-1 14, 2014

WOLFF, Annika; ZDRAHAL, Zdenek; NIKOLOV, Andriy; PANTUCEK, Michal. Improving retention: predicting at-risk students by analysing clicking behaviour in a virtual learning environment. INTERNATIONAL CONFERENCE ON LEARNING ANALYTICS AND KNOWLEDGE, 3, 2013, Leuven. Proceedings... Leuven, 2013.

26 WOODLEY, Alan; LANGE, Paul; TANEWSKI, George. Student progress in distance education: Kember's model re-visited. Open Learning, Milton Keynes, v. 16, n. 2, p. $113-131$, 2001

WOODLEY, Alan; PARLETT, Malcom. Student drop-out. Teaching at a Distance, Milton Keynes, n. 24, p. 2-23, 1983.

WYATT, Linda. Nontraditional student engagement: Increasing adult student success and retention. The Journal of Continuing Higher Education, Abingdon, v. 59, n. 1, p. 10-20, 2011.

XENOS, Michalis; PIERRAKEAS, Christos; PINTELAS, Panagiotis. A survey on student dropout rates and dropout causes concerning the students in the Course of Informatics of the Hellenic Open University. Computers and Education, Amesterdã, v. 39, n. 4, p. 361-377, 2002.

YASMIN. Application of the classification tree model in predicting learner dropout behaviour in open and distance learning. Distance Education, Melbourne, v. 34, n. 2, p. 218-231, 2013.

ZERBINI, Thaís. Avaliação da transferência de treinamento em curso a distância. 2007. 32 1f. Tese - Instituto de Psicologia, Universidade de Brasília, Brasília, 2007. 
Estratégias de aprendizagem, reações aos procedimentos de um curso via internet, reações ao tutor e impacto do treinamento no trabalho. 2003. 21 Of. Dissertação - Instituto de Psicologia, Universidade de Brasília, Brasília, 2003.

ZERBINI, Thaís; ABBAD, Gardênia. Aprendizagem induzida pela instrução em contexto de organizações e trabalho: uma análise crítica da literatura. Cadernos de Psicologia Social do Trabalho, São Paulo, v. 13, n. 2, p. 177-193, 2010.

Ms. Oberdan Santos da Costa Universidade Fernando Pessoa Faculdade de Ciência e Tecnologia, Porto (Portugal) Grupo de Pesquisa TRS - Grupo de Tecnologia, Rede e Sociedade E-mail: sc.oberda@gmail.com

Prof. Phd. Luis Borges Gouveia Universidade Fernando Pessoa

Faculdade de Ciência e Tecnologia, Porto (Portugal) Grupo de Pesquisa TRS, Grupo de Tecnologia, Rede e Sociedade. E-mail:Imbg@ufp.edu.pt Recebido 3 out. 2018 Aceito 30 nov. 2018 